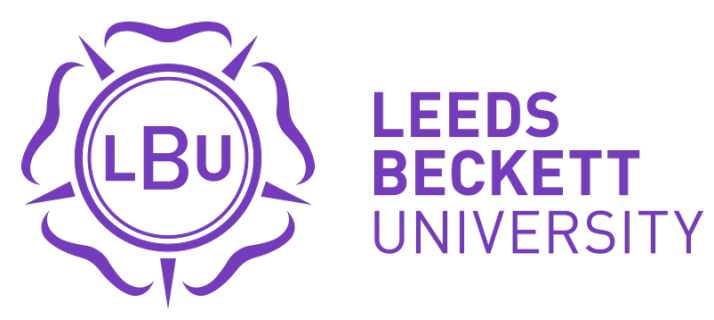

Citation:

Brierley, C and Atwell, E (2009) Exploring complex vowels as phrase break correlates in a corpus of English speech with ProPOSEL, a prosody and POS English lexicon. Proceedings of the Annual Conference of the International Speech Communication Association, INTERSPEECH. 868 - 871.

Link to Leeds Beckett Repository record:

https://eprints.leedsbeckett.ac.uk/id/eprint/2137/

Document Version:

Article (Accepted Version)

The aim of the Leeds Beckett Repository is to provide open access to our research, as required by funder policies and permitted by publishers and copyright law.

The Leeds Beckett repository holds a wide range of publications, each of which has been checked for copyright and the relevant embargo period has been applied by the Research Services team.

We operate on a standard take-down policy. If you are the author or publisher of an output and you would like it removed from the repository, please contact us and we will investigate on a case-by-case basis.

Each thesis in the repository has been cleared where necessary by the author for third party copyright. If you would like a thesis to be removed from the repository or believe there is an issue with copyright, please contact us on openaccess@leedsbeckett.ac.uk and we will investigate on a case-by-case basis. 


\title{
Exploring Complex Vowels as Phrase Break Correlates in a Corpus of English Speech with ProPOSEL, a Prosody and POS English Lexicon.
}

\author{
Claire Brierley $^{12}$, Eric Atwell ${ }^{2}$ \\ ${ }^{1}$ School of Games Computing and Creative Technologies, University of Bolton, UK \\ ${ }^{2}$ School of Computing, University of Leeds, UK \\ cb5@bolton.ac.uk, eric@comp.leeds.ac.uk
}

\begin{abstract}
Real-world knowledge of syntax is seen as integral to the machine learning task of phrase break prediction but there is a deficiency of a priori knowledge of prosody in both rule-based and data-driven classifiers. Speech recognition has established that pauses affect vowel duration in preceding words. Based on the observation that complex vowels occur at rhythmic junctures in poetry, we run significance tests on a sample of transcribed, contemporary British English speech and find a statistically significant correlation between complex vowels and phrase breaks. The experiment depends on automatic text annotation via ProPOSEL, a prosody and part-of-speech English lexicon.
\end{abstract}

Index Terms: prosody; real-world knowledge for machine learning; phrase break prediction; text-to-speech synthesis

\section{Introduction}

The goal of automatic phrase break prediction is to identify prosodic-syntactic boundaries in any given text which, on human evaluation, constitute natural and intelligible phrasing, and which can confidently be used as input features to a speech synthesizer for modelling intonation and duration over chunks of text designated by these boundaries. Traditionally, the phrase break classifier is trained on a speech corpus with gold standard part-of-speech (PoS) and boundary annotations and tested on an unseen reference dataset from the same corpus; its task is to recapture original boundary locations stripped from the test set by classifying tokens in the input text as either breaks or non-breaks.

Real-world knowledge of syntax is seen as integral to this machine learning task but there is a deficiency of a priori knowledge of prosody in both rule-based and data-driven classifiers. We therefore explore prosodic features in the form of complex vowels as potential phrase break correlates, based on the observation that complex vowels tend to occur at rhythmic junctures in poetry.

In a previous paper [1], we have discussed machinelearning techniques and evaluation metrics used in phrase break prediction, plus the inherent problem of prosodic variance: more than one natural and intelligible phrasing (i.e. more than one gold standard) exists for most sentences; and models trained on one corpus may not generalise to other domains. Here we begin with an overview of features and feature sets used when predicting boundaries, before hypothesizing and testing non-traditional, vocalic phrase break correlates in a sample from the Aix-MARSEC corpus of English speech [2] via the chi-squared test for independence. This entails automatic annotation of the dataset with domain knowledge from ProPOSEL, a prosody and syntax English lexicon [3], [4].

\section{Features used in phrase break prediction}

Syntactic features are integral to phrase break prediction because of the overlap between syntactic and prosodic phrasing. The boundary annotation $/ / /$ in the following sentence taken from a landmark psycholinguistic study [5], represents human consensus on the best place to pause:

After the cold winter of that year I most people were totally fed-up.

The least sensitive and most transferable syntactic feature for predicting phrase breaks is content-function word status. Under this rule-based scheme, boundaries are inserted after punctuation and between open-class content words or chunks and closed-class function words or chinks [6].

For our model sentence, function-word groups captured by a standard CFP algorithm match syntactic units delineated by the Link parser [7]:

$\begin{array}{ll}\text { PP } & \text { After the cold winter } \\ \text { PP } & \text { of that year } \\ \text { NP } & \text { most people } \\ \text { VP } & \text { were totally fed-up }\end{array}$

Edinburgh's Festival speech synthesis system implements a stochastic model for phrase break prediction which requires more discrete syntactic information from partof-speech (PoS) tags.

After_CTS the AT0 cold_AJ0 winter_NN1 of_PRF that_DT0 year_NN1 most_DTO people_NNO were_VBD totally_AVO fed-up_AJO...

Our sample sentence is annotated with the British National Corpus C5 PoS tag set [23]

The Festival classifier integrates two feature sets: localised observation probabilities of PoS trigrams given juncture type, conditioned on long-distance syntactic information from a high-order n-gram juncture sequence model [8].

Building on the intuition that phrase breaks occur between major syntactic units $\{\mathrm{NP}$; VP; PP; ADJP; ADVP $\}$, Koehn et al., (2000) use a sophisticated feature set [9] incorporating binary flags for whether or not the token initiates a major phrase or sub-clause. Their impressive prediction rate of $90.8 \%$ for boundary detection is partly accounted for by their incorporation of a feature derived from hand-labelled transcriptions: i.e. accent status of words adjacent to the boundary site; whereas the aim is to predict prosodic events like phrase breaks and accents automatically.

Taylor and Black [8], and more recently Ingulfsen et al. [10], have demonstrated that punctuation is the single most important source of information for phrase break classification, finding approximately $50 \%$ of all breaks. Other text-based features which have been used to supplement syntactic features, include: word counts denoting length of utterance and distance of potential boundary site from start and 
end of sentence [11]; total number of words and syllables, plus distance from start and finish of utterance in words, syllables and stressed syllables, plus distance of potential boundary site from last punctuation mark [9], [12].

Recent work [10], [13] revisits syntactic features to determine the effectiveness of deep versus shallow linguistic representations for phrase break prediction. The best performing models in these studies use a combined set of long-range parse features and shallow representations incorporating different levels of granularity: CFP tags and PoS trigrams.

Non-traditional features in the form of syllable counts have previously been implemented in syntax-based phrase break models for English to regulate the number of syllables in any one intonational phrase [14]; and as a distance metric for encoding global information in the sentence [15]. A recent study by Ananthakrishnan and Narayanan [16] attempts to integrate the prediction of accents and boundaries based on combined feature streams (acoustic, lexical and syntactic) and finds that lexical syllable tokens, augmented with canonical stress labels derived from an open source pronunciation lexicon, are effective for accent detection but not for boundary prediction.

\section{Hypothesizing non-traditional phrase break correlates}

Ananthakrishnan and Narayanan conclude that syllable tokens are poorer indicators of boundary events than PoS tags. However, this conclusion is based only on word-final syllable tokens minus stress weightings for the phrase break prediction task; word-initial and medial syllables are automatically classed as non-breaks because they are never immediately followed by boundary tokens.

We wish to question the assumption that non word-final syllabic nuclei (e.g. the second syllable in seCURity) have no influence on boundary placement and to test the hypothesis that complex vowels - i.e. diphthongs and triphthongs - might emerge as useful predictive features for phrase break models, irrespective of where they occur within a word. There is consensus within the ASR research community that pauses affect vowel durations in preceding words [17]. We wish to reverse the perspective on prepausal lengthening and ask to what extent a domain-independent feature like complex vowels may be said to induce boundaries.

The intuition that the presence of complex vowels in (content) words increases the likelihood of their being classified as breaks comes from poetry [18], where diphthongs and triphthongs seem to be associated with rhythmic junctures. This happens within lines and across lines as in Blake's The Tyger (circa 1794):

Tyger! Tyger! | burning bright |

In the forests | of the night |

\section{Leveraging real-world knowledge of prosody from the lexicon}

One of the thematic programmes for PASCAL-2 (2008) identifies a current interest in, and trend towards, leveraging real-world knowledge to enhance performance in machine learning in a variety of application domains, including text and language processing, where previously little a priori knowledge has been assumed on the part of the learning mechanism. Our survey reveals a deficiency of a priori linguistic knowledge of prosody in the feature sets typically used in rule-based and data-driven phrase break models. In contrast, a competent human reader is able to project holistic linguistic insights, including projected prosody, onto text and to treat them as part of the input [19]. It is our contention that human readers may use the sound patterns inherent in complex vowels as linguistic signs for phrase breaks in as yet undefined contexts. Such signs can be extracted from the lexicon and presented as input features for the phrase break classifier in the same way that real-world knowledge of syntax is represented in PoS tags.

\subsection{ProPOSEL: a prosody and PoS English lexicon}

ProPOSEL [3], [4] is a prosody and PoS English lexicon derived from several widely-used lexical resources for computer speech and language. ProPOSEL's multi-field format classifies 104049 word forms under four variant PoStagging schemes mapped to default closed and open-class word categories; plus canonical phonetic transcriptions; syllable counts; consonant-vowel (CV) patterns; and abstract representations of rhythmic structure or canonical stress labels. An example entry group for the verb secure is given in Table 1 .

\begin{tabular}{|l|l|l|l|}
\hline Field & Sample & Field & Sample \\
\hline $\mathbf{1}$ wordform & secure & $\begin{array}{l}\mathbf{9} \text { Penn Treebank } \\
\text { tag }\end{array}$ & VB \\
\hline $\mathbf{2}$ C5 tag & VVI & $\begin{array}{l}\mathbf{1 0} \text { content or } \\
\text { function word tag }\end{array}$ & C \\
\hline $\begin{array}{l}\mathbf{3} \text { Capitalisation } \\
\text { flag }\end{array}$ & 0 & $\mathbf{1 1 ~ L O B ~ t a g}$ & VB \\
\hline $\mathbf{4}$ SAM-PA & sl'kjU@R & $\mathbf{1 2 ~ C 7 ~ t a g ~}$ & VVI \\
\hline $\begin{array}{l}\mathbf{5} \text { CUV2 tag \& } \\
\text { frequency rating }\end{array}$ & H2\%,OA\% & $\begin{array}{l}\mathbf{1 3 ~ D I S C ~ s y l l a b i f i e d ~} \\
\text { transcription }\end{array}$ & sl-'kj9R \\
\hline $\begin{array}{l}\mathbf{6} \text { C5 tag \& BNC } \\
\text { frequency rating }\end{array}$ & VVI:25 & $\begin{array}{l}\mathbf{1 4} \text { DISC syllable- } \\
\text { stress mapping }\end{array}$ & sl:0 'kj9R:1 \\
\hline $\mathbf{7}$ syllable count & 2 & $\mathbf{1 5}$ CV pattern & [CV][CCVVC] \\
\hline $\begin{array}{l}\mathbf{8} \text { lexical stress } \\
\text { pattern }\end{array}$ & 01 & & \\
\hline
\end{tabular}

Table 1: ProPOSEL's 15 pipe-separated fields constitute a purposebuilt repository of linguistic concepts in accessible text file format.

To investigate the correlation between complex vowels and phrase breaks, we have automatically tagged an extract from the Aix-MARSEC corpus with shallow parse features and canonical phonetic transcriptions from ProPOSEL, and run a chi-squared test to determine whether this correlation is statistically significant or not. We have used the same development sets as in previous studies [1], [20]: a BBC radio recording from the 1980s of a Reith lecture in Section C of the corpus, with illustrative examples drawn from sections A08 and A09: informal news commentaries.

Preparing the dataset prior to dictionary lookup was nontrivial and involved several stages. The first task was to map annotation tiers in overlapping subfiles in the Aix-MARSEC sample in order to label each word as a break or non-break (\$4.2). Word and phrase break classifications in AixMARSEC were then merged with corresponding PoS-tagged text in the Spoken English Corpus [21], discrepancies intervene: compounds and abbreviations are handled differently in both datasets, for example (\$4.3). Next, the corpus was re-tagged with the PoS tag scheme used in the lexicon i.e. a discriminating tagset (LOB) was collapsed into a sparser one (C5) (§4.4). Finally, desired information from the lexicon was projected onto the dataset by matching up wordC5 pairings ( $\$ 4.5)$. 


\subsection{Mapping tiers in Aix-MARSEC}

The Aix-MARSEC Corpus has multi-level prosodic annotation tiers aligned with the speech signal; the two tiers used in this study are for plain text plus intonation units (IUs) delineated by phrase break mark-up / / /. The SAMP-PA transcriptions from the syllables tier were not used in our study because we are interested in predictive features derived from speaker-independent and domain-independent citation forms in ProPOSEL which can be superimposed on any unseen English text - for example, seventeenth century English verse $c f$. [18].

Each section in Aix-MARSEC is split up into a series of much smaller, overlapping TextGrid files. Merging the text and IUs tiers was therefore accomplished on a file-by-file basis, using interval tokens to retrieve a match between tiers. The resulting list objects were concatenated in a final list listAllText - ready for merger with the corresponding file in the Spoken English Corpus (SEC) to capture PoS-tags.

\subsection{Merging Aix-MARSEC and SEC files}

The target data structure for dictionary lookup ( $\$ 4.5)$ is a nested list where each index holds values for: word token; break class; punctuation; and PoS-tag. Capturing PoS tags from SEC entailed looping over two parallel lists of unequal length - listAllText and a list of word_PoS pairings from SEC - a process complicated by the fact that compound words are represented differently in both datasets, and furthermore, that punctuation in SEC does not always correspond to boundaries or placeholders in Aix-MARSEC. Such problems are exemplified in Listing 1 (section A09 of the corpus), where we find different representations for the compound adjective: cross-ethnic; variant phrasing for the fragment: who two years ago; no apparent placeholder in Aix-MARSEC following the boundary after ago; no punctuation in SEC after the word together, which is marked as a phrase break in Aix-MARSEC.

\begin{tabular}{|c|c|}
\hline Aix-MARSEC & SEC \\
\hline $\begin{array}{l}\text { ['ethnic', '48.69', 'I'] } \\
\text { ['\#', '48.74', 'P'] } \\
\text { ['cross', '49.12', 'non-break'] } \\
\text { ['ethnic', '49.53', 'I'] } \\
\text { ['\#', '49.62', 'P'] } \\
\text { ['and', '49.88', 'non-break'] } \\
\text { ['political', '50.41', 'non-break'] } \\
\text { ['parties', '50.88', 'I'] } \\
\text { ['\#', '51.39', 'P'] } \\
\text { ['who', '51.59', 'non-break'] } \\
\text { ['two', '51.73', 'non-break'] } \\
\text { ['years', '52.04', 'non-break'] } \\
\text { ['ago', '52.44', 'I'] } \\
\text { ['came', '52.70', 'non-break'] } \\
\text { ['together', '53.12', 'I'] } \\
\text { ['\#',' '53.17', 'P'] } \\
\text { ['to', '53.34', 'non-break'] }\end{array}$ & $\begin{array}{l}\text { JJ ethnic } \\
\text { 'JJ ' cross-ethnic } \\
\text { ' CC' and } \\
\text { JJ political } \\
\text { ' ' } \\
\text { NNS parties } \\
\text { WP who } \\
\text { ' ' } \\
\text { CD two } \\
\text { NNS years } \\
\text { RB ago } \\
\text { ' ' } \\
\text { VBD came } \\
\text { RB together } \\
\text { TO to }\end{array}$ \\
\hline
\end{tabular}

Listing 1: Transcriptions of the same utterance in two different versions of the corpus exhibit variant phrasing.

\subsection{Mapping between PoS tag sets using ProPOSEL}

List indices in the object listAllText have now acquired PoS tags and, if present, punctuation from the semi-automatic process just described. However, the recommended lookup strategy with the prosody and PoS lexicon is via compound dictionary keys comprising word_C5 pairings. A range of tagsets (Penn, LOB and C7) were mapped to C5 as part of lexicon build; and ProPOSEL's software tools provide solutions for mapping between schemes (Brierley and Atwell, 2008a). In the present study, a more discriminating tagset -
LOB [22] - is collapsed into a sparser scheme (C5). As part of this process, enclitics in LOB are re-formatted in a style compatible with the lexicon; instances such as: ['BEDZ', 'was', '>', 'XNOT', "n't", '<'] and ['WP', 'who', '>', 'HV', "'ve", '<'] are transformed into: ['BEDZ+XNOT', "wasn't"] and ['WP+HV', "who've"].

\subsection{Dictionary lookup and text annotation}

Nested arrays in listAllText are finally augmented with domain knowledge of prosody (e.g. DISC fields in ProPOSEL) and coarse-grained syntactic information (default content-function word tags) via intersection with ProPOSEL. Listing 2 first builds an instance of the dictionary object proPOSEL with compound keys word_C5 tuples mapped to selected values. Python's itertools() module is then used to loop through two parallel iterables: listAllText and match, a sequence of word_C5 tuples from the same dataset. Items in the latter are compared against ProPOSEL's keys; a successful match appends dictionary values associated with those keys to the parallel nested position in listAllText.

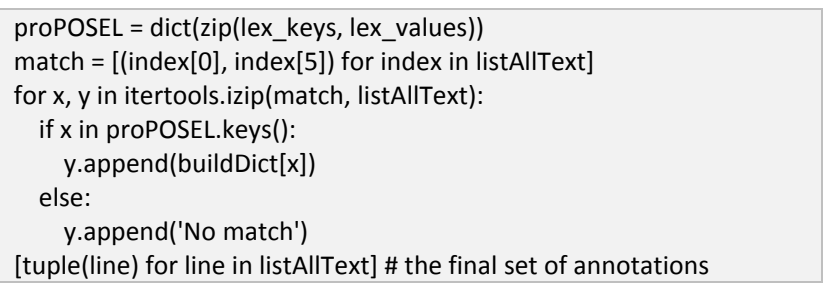

Listing 2: Intersection between the dictionary object proPOSEL and the sequence object match appends dictionary values to the parallel position in listAllText.

Inner lists in listAllText have now been augmented with content/function-word tags, DISC phonetic transcriptions and canonical stress weightings aligned with syllables (e.g. the lexical stress pattern 2010 assigned to the DISC transcription for the word contribution: "kQn:2 trl:0 \'bju:1 SH:0).

\section{Significance Testing}

Each word in the sample was assigned to one of four different categories and counts for each category were entered in a $2 \times 2$ contingency table (Table 2) ready for the chi-square test. The category label of diphthongs is used here to denote all complex vowels. The total word count is simply the length of listAllText minus the count for unmatched items; these were not included in the final calculation and figures used in Table 2 reflect this.

\begin{tabular}{|l|r|r|r|}
\hline \multirow{2}{*}{ GROUPS } & \multicolumn{2}{|c|}{ OUTCOMES } & \\
\cline { 2 - 4 } & \multicolumn{1}{|c|}{ Breaks } & Non-breaks & \\
\hline Diphthongs & $\mathbf{2 0 1}$ & 298 & $\mathbf{4 9 9}$ \\
\hline No diphthongs & 437 & 1357 & 1794 \\
\hline & $\mathbf{6 3 8}$ & 1655 & $\mathbf{2 2 9 3}$ \\
& $(696-58)$ & & $(2468-175)$ \\
\hline
\end{tabular}

Table 2: A 2 × 2 contingency table records the observed frequency distribution for target groups and outcomes from the corpus sample.

The chi-square test in this experiment determines whether the distribution resulting from observed frequencies in the shaded area in Table 2 is significantly different from the chance distribution anticipated from expected frequencies. The latter are calculated via marginal totals for rows and columns in the table: for example, the expected frequency for diphthongs classified as breaks is given by $(638 / 2293) * 499$. Table 3 presents observed versus expected frequencies (given in bold and expressed as whole numbers for clarity of presentation) for all four categories. 


\begin{tabular}{|c|c|c|}
\hline \multirow[t]{2}{*}{ GROUPS } & \multicolumn{2}{|c|}{ OUTCOMES } \\
\hline & Breaks & Non-breaks \\
\hline Diphthongs & 201 & 298 \\
\hline & 127 & 360 \\
\hline No diphthongs & 437 & 1357 \\
\hline
\end{tabular}

Table 3: Observed and expected frequencies are used to find the value of $\chi^{2}$ in this test for independence.

These figures are then used to find the value of $\chi^{2}$ according to the formula:

$$
\chi^{2}=\sum \frac{\left(f_{o}-f_{e}\right)^{2}}{f_{e}}
$$

The null hypothesis $H_{o}$ assumes that the distributions will be the same or that the difference will not exceed some critical value. In our case, however, $H_{o}$ can be rejected because the association between groups and outcomes turns out to be extremely statistically significant: chi squared equals 49.28 , with one degree of freedom, and a two -tailed p-value which is less than 0.0001 . This p-value represents the odds ratio for achieving the same result through random sampling. Finally, since there are only four diphthong-bearing function words which are also classified as breaks in this sample, we can hypothesize that the significant correlation is actually between diphthong-bearing content words and phrase breaks.

\section{Conclusion}

Our survey of features used in phrase break prediction highlights a deficiency of a priori knowledge of prosody in both rule-based and data-driven language models. The authors concur with studies that recognise how, even in silent reading, humans project prosody onto text and treat it as part of the input. Hence we have developed ProPOSEL, a domainindependent lexical resource and prosodic-syntactic text annotation tool.

There is consensus in the ASR community that pauses affect vowel durations in adjacent words. Based on intuitions from poetry and concurrent work [18], have redefined this causal relationship and interpreted complex vowels as phrase break signifiers. From significance tests on a sample of contemporary British English speech from the Aix-MARSEC Corpus, plus seventeenth century English verse (ibid.), we now have empirical evidence that diphthong-bearing content words are highly correlated with phrase breaks.

Since accent status of pre-boundary words has already proved effective in phrase break prediction, future work will focus on the correlation of complex vowels, salient pitch accents and boundaries to explore a linguistically-motivated hypothesis: native English speakers subconsciously favour diphthong-bearing words as tonics (i.e. nuclear prominences in tone groups) because vocalic glides facilitate sudden pitch transitions between high and low tones: the hallmark of salient pitch accents.

\section{References}

[1] Brierley, C. and Atwell, E., "Prosodic Phrase Break Prediction: Problems in the Evaluation of Models against a Gold Standard", in Traitement Automatique des Langues, 48(1):187-206, 2007b.

[2] Auran, C., Bouzon, C. and Hirst, D., "The Aix-MARSEC Project: an Evolutive Database of Spoken British English", in Proc. Speech Prosody (SP-2004), 561-564, 2004.

[3] Brierley, C. and Atwell, E., "ProPOSEL: A Prosody and POS English Lexicon for Language Engineering" in Proc. $6^{\text {th }}$ Language Resources and Evaluation Conference (LREC 2008), 2008a.
[4] Brierley, C. and Atwell, E., "A Human-oriented Prosody and PoS English Lexicon for Machine Learning and NLP' in Proc. $22^{\text {nd }}$ International Conference on Computational Linguistics (Coling 2008), Workshop on Cognitive Aspects of the Lexicon, 2008b.

[5] Gee, J. P., Grosjean, F., "Performance Structures: A Psycholinguistic and Linguistic Appraisal", in Cognitive Psychology, (15), 411-458, 1983.

[6] Liberman, M. Y. and Church, K. W., "Text Analysis and Word Pronunciation in Text-to-Speech Synthesis", in Furui, S. and Sondhi, M. M. (eds.) Advances in Speech Signal Processing New York, Marcel Dekker, Inc., 1992.

[7] Temperley, D., Sleator, D. and Lafferty, J., "Link Grammar", Online: http://www.link.cs.cmu.edu/link/, accessed on 15 July 2009.

[8] Taylor, P. and Black, A. W., "Assigning Phrase Breaks from Part-of-Speech Sequences" in Computer Speech and Language, 12(2):99-117, 1998

[9] Hirschberg J. and Prieto P., "Training Intonational Phrasing Rules Automatically for English and Spanish Text-to-speech", in Speech Communication, 18(3):281-290, 1996.

[10] Ingulfsen, T., Burrows, T. and Buchholz, S., "Influence of Syntax on Prosodic Boundary Prediction", in Proc. INTERSPEECH 2005, 1817-1820, 2005.

[11] Wang, M. Q. and Hirschberg J., "Predicting Intonational Phrasing from Text", in Proc. Association for Computational Linguistics (ACL 1991), 285-292, 1991.

[12] Koehn P., Abney, S., Hirschberg, J. and Collins, M., "Improving Intonational Phrasing with Syntactic Information", in Proc. IEEE International Conference on Acoustics, Speech, and Signal Processing (ICASSP), 3:1289-1290, 2000.

[13] Read, I. and Cox, S., "Stochastic and Syntactic Techniques for Predicting phrase Breaks", in Computer Speech and Language, 21(3):519-542, 2007.

[14] Atterer, M. and Klein, E., "Integrating Linguistic and Performance-Based Constraints for Assigning Phrase Breaks" in Proc. $19^{\text {th }}$ International Conference on Computational Linguistics (Coling 2002), 29-35, 2002.

[15] Schmid, H. and Atterer, M., "New Statistical Methods for Phrase Break Prediction", in Proc. $20^{\text {th }}$ International Conference on Computational Linguistics (Coling 2004), 659-665, 2004.

[16] Ananthakrishnan, S. and Narayanan, S.S., "Automatic Prosodic Event Detection Using Acoustic, Lexical, and Syntactic Evidence", in IEEE Transactions on Audio, Speech, and Language Processing (TASLP 2008), 16(1):216-228, 2008.

[17] Vergyri, D., Stolcke, A., Gadde, V.R.R., Ferrer, L. and Shriberg, E., "Prosodic Knowledge Sources for Automatic Speech Recognition", in Proc. International Conference on Acoustics, Speech and Signal Processing (ICASSP 2003), 208-211, 2003.

[18] Brierley, C. and Atwell, E., "Holy Smoke: Vocalic Precursors of Phrase Breaks in Milton's Paradise Lost", submitted to Literary and Linguistic Computing, 2009.

[19] Fodor, J. D., "Psycholinguistics Cannot Escape Prosody", in Proc. Speech Prosody (SP-2002), 83-90, 2002.

[20] Brierley, C. and Atwell, E., "An Approach for Detecting Prosodic Phrase Boundaries in Spoken English", in ACM Crossroads Journal, $14(1), \quad$ Online: http://www.acm.org/crossroads/xrds14-1/nltklite.html, accessed 15 July 2009

[21] Taylor, L. J. and Knowles, G., "Manual of information to accompany the SEC corpus: The machine readable corpus of spoken English", UCREL, University of Lancaster, 1988.

[22] Johansson, S., Atwell, E., Garside, R. and Leech, G., "The Tagged LOB Corpus Users' Manual, Online: http://khnt.hit.uib.no/icame/manuals/lobman/index.htm, accessed 15 July 2009

[23] Leech, G and Smith, N., "Manual to accompany the British National Corpus (Version 2) with Improved Word-class Tagging", http://www.natcorp.ox.ac.uk/docs/bnc2postag_manual.htm, accessed 15 July 2009. 